Artículo

\title{
Biomonitoreo de metales pesados en vegetación arbórea en la ciudad de Saltillo
}

\author{
Eduardo Ramírez Méndez ${ }^{1}$ \\ Jesús Valdés-Reyna ${ }^{1 \S}$ \\ Antonio Juárez-Maldonado ${ }^{2}$ \\ Nadia Valentina Martínez-Villegas ${ }^{3}$ \\ Lorenzo Alejandro López-Barbosa ${ }^{4}$
}

${ }^{1}$ Especialidad en Manejo Sustentable de Zonas Áridas y Semiáridas. ${ }^{2}$ Departamento de BotánicaUniversidad Autónoma Agraria Antonio Narro. (juma841025@gmail.com). ${ }^{3}$ Instituto Potosino de Investigación Científica y Tecnológica, AC. (nadia.martinez@ipicyt.edu.mx). ${ }^{4}$ Departamento de Sociología-Universidad Autónoma Agraria Antonio Narro. (lalopbar@yahoo.com.mx).

${ }^{\S}$ Autor para correspondencia: jesus.valdes@ uaaan.edu.mx.

\section{Resumen}

La contaminación con metales pesados es un problema grave a nivel mundial y en algunas regiones de México. En las ciudades, esto se debe principalmente a residuos o desechos de la industria y al flujo vehicular principalmente. El presente estudio está enfocado en determinar la concentración de metales pesados $(\mathrm{Cd}, \mathrm{Cr}, \mathrm{Pb}$ y $\mathrm{V}$ ) en hojas de especies arbóreas (Pinus halepensis Mill., Cupressus sempervirens L. y Prosopis spp.). Estas especies fueron seleccionadas con la finalidad de ser utilizadas como bioindicadoras de la calidad ambiental en la ciudad de Saltillo, Coahuila, México. Se establecieron 15 puntos de muestreo, de los cuales 13 puntos fueron distribuidos aleatoriamente en la ciudad de Saltillo y dos puntos fuera de la mancha urbana. El muestreo se realizó en el mes de julio de 2019 (época del verano). El contenido de metales pesados se determinó mediante la técnica de espectrometría de emisión óptica de plasma inductivamente acoplado (ICPOES). Se probaron las interacciones entre los factores especie-sitio con respecto a las concentraciones de metales pesados, mismas que fueron determinadas a través de los promedios obtenidos entre las muestras y sus duplicados. Destaca que el factor especie fue $C$. sempervirens resulto la de mayor concentración de metales pesados en $\mathrm{Cd}, \mathrm{Pb}$ y $\mathrm{V}$, así como P. halepensis en Cr. Los puntos 3, 4, 12 y 15 resultaron con las mayores concentraciones de los metales analizados, dichos puntos se encuentran en las zonas sureste y céntrica de la ciudad de Saltillo. El uso de especies arbóreas como bioindicadoras de metales pesados en la ciudad de Saltillo puede ser una herramienta viable para el monitoreo de la calidad ambiental.

Palabras clave: bioindicador, contaminación, desarrollo urbano, hojas, residuos de la industria.

Recibido: abril de 2021

Aceptado: junio de 2021 


\section{Introducción}

El acelerado crecimiento de los sistemas urbanos e industriales ha conllevado a diversos impactos ambientales (Rodríguez et al., 2017), como lo es la creciente emisión al ambiente de sustancias contaminantes provenientes de la industria, como la liberación de cantidades masivas de dióxido de carbono, óxidos de azufre y nitrógeno, polvo, partículas suspendidas totales y sustancias químicas tóxicas (Henry et al., 1999).

Entre los principales factores de esta problemática se encuentran las actividades económicas del lugar, la infraestructura de transporte y movilidad, así como también las características fisiográficas de la región (SEMA, 2017). Entre las partículas suspendidas totales (PST), se pueden encontrar contaminación atmosférica por metales pesados (MP) en las ciudades, tales como $\mathrm{Pb}, \mathrm{Cd}, \mathrm{Ni}, \mathrm{Cu}$, Cr (Fernández et al., 2000; Cruz et al., 2013), así como Zn, Sb, Ba dependiendo el tipo de industria que se desarrolle en la región (Rodríguez et al., 2017).

Las principales fuentes de contaminación por MP son la minería, la metalúrgica, la agricultura, los vehículos automotores, entre otros (Covarrubias y Cabriales, 2017). Los MP constituyen un grupo de elementos con características físicas, químicas y biológicas muy heterogéneas (González et al., 2017). Se consideran MP a aquellos elementos constituidos con una densidad mayor a $5 \mathrm{~g} \mathrm{~cm}^{-3}$, se clasifican en esenciales y no esenciales, estos últimos no presentan función biológica y su presencia puede ser tóxica, mientras que los esenciales son requeridos por todos los organismos en cantidades trazas (González et al., 2017).

Los MP se encuentran generalmente como componentes naturales de la corteza terrestre, en forma de minerales, sales u otros compuestos, estos no pueden ser degradados o destruidos fácilmente de forma natural o biológica ya que no tienen funciones metabólicas específicas para los seres vivos (Hernández et al., 2017). Los MP, se encuentran generalmente en bajas concentraciones en el ambiente, aunque, como resultado de las actividades antropogénicas, sus niveles se han incrementado (Liu et al., 2018), ocasionando contaminación en los ecosistemas expuestos a estos.

Estos MP son riesgosos porqué están ligados a una serie de problemas en la salud humana como cáncer gastrointestinal, daños al sistema inmunológico, daños de tipo mental y malnutrición, entre otros (Kumar et al., 2019). Para la rehabilitación o restauración de los ecosistemas por contaminación de MP, es necesario conocer su destino, así como la intensidad, ya que pueden implementarse alternativas de uso de métodos biológicos particulares, esto da lugar a que en las ciudades se pueda utilizar la vegetación como indicadores biológicos o como monitores de contaminantes específicos (Turkyilmaz et al., 2018).

El desarrollo urbano en la ciudad de Saltillo se ha incrementado considerablemente, cuenta con el mayor número de habitantes (807 537), mayor número de viviendas particulares habitadas (213 329) y la tasa más alta de crecimiento promedio anual $(2.3 \%)$ del estado de Coahuila (INEGI, 2015). En el municipio se observa la expansión industrial, entre las que destacan, las de ensamblado automovilístico, de autopartes y manufacturero (Mendoza, 2001), así como las actividades comerciales y de servicios que se desarrollan en la ciudad, lo que ha conllevado a un incremento en la contaminación ambiental del municipio (SEMA, 2017). 
El inventario de emisiones de contaminantes a la atmósfera es un instrumento estratégico para la gestión de la calidad del aire que permite conocer el tipo y cantidad de contaminantes que son emitidos al aire por los diferentes sectores o categorías. Así se establece un monitoreo de calidad del aire del municipio de Saltillo por parte de la SEMA en el año 2017, en donde los principales contaminantes antropogénicos por PM10 son caminos no pavimentados $63.14 \%$, caminos pavimentados $9.34 \%$, metalúrgica (incluye la siderúrgica) $8.8 \%$, ladrilleras $4.47 \%$, vehículo> 3 t y tractocamiones $3.02 \%$, industria metálica $2.94 \%$ y de las PM 2.5 en caminos no pavimentados $39.63 \%$, metalúrgica (incluye la siderúrgica) $16.98 \%$, ladrilleras $10.71 \%$, vehículo $>3$ t y tracto camiones $7.28 \%$, caminos pavimentados $5.86 \%$ y la industria metálica 5.72\% (SEMA, 2017). El objetivo del presente trabajo consistió en analizar la concentración de metales pesados $(\mathrm{Cd}, \mathrm{Cr}, \mathrm{Pb}$ y V) en las hojas de especies arbóreas en la ciudad; determinar sus concentraciones y compáralos con límites de toxicidad en especies vegetales, así como determinar el área de mayor concentración en la ciudad de Saltillo, Coahuila.

\section{Materiales y métodos}

El estudio se llevó a cabo en la ciudad de Saltillo, donde se realizó la toma de muestras de diferentes puntos distribuidos de acuerdo con la estructura de la ciudad buscando que hubiera aproximadamente $1 \mathrm{~km}$ de distancia entre cada punto. Se establecieron 15 puntos de muestreo, de los cuales 13 fueron distribuidos dentro de la ciudad de Saltillo y dos puntos fuera de la mancha urbana considerados como control (Figura 1). Las especies vegetales muestreadas son las especies arbóreas más representativas en la ciudad y fueron Pinus halepensis, Cupressus sempervirens y Prosopis glandulosa, las cuales mostraron tener una amplia distribución en la ciudad.

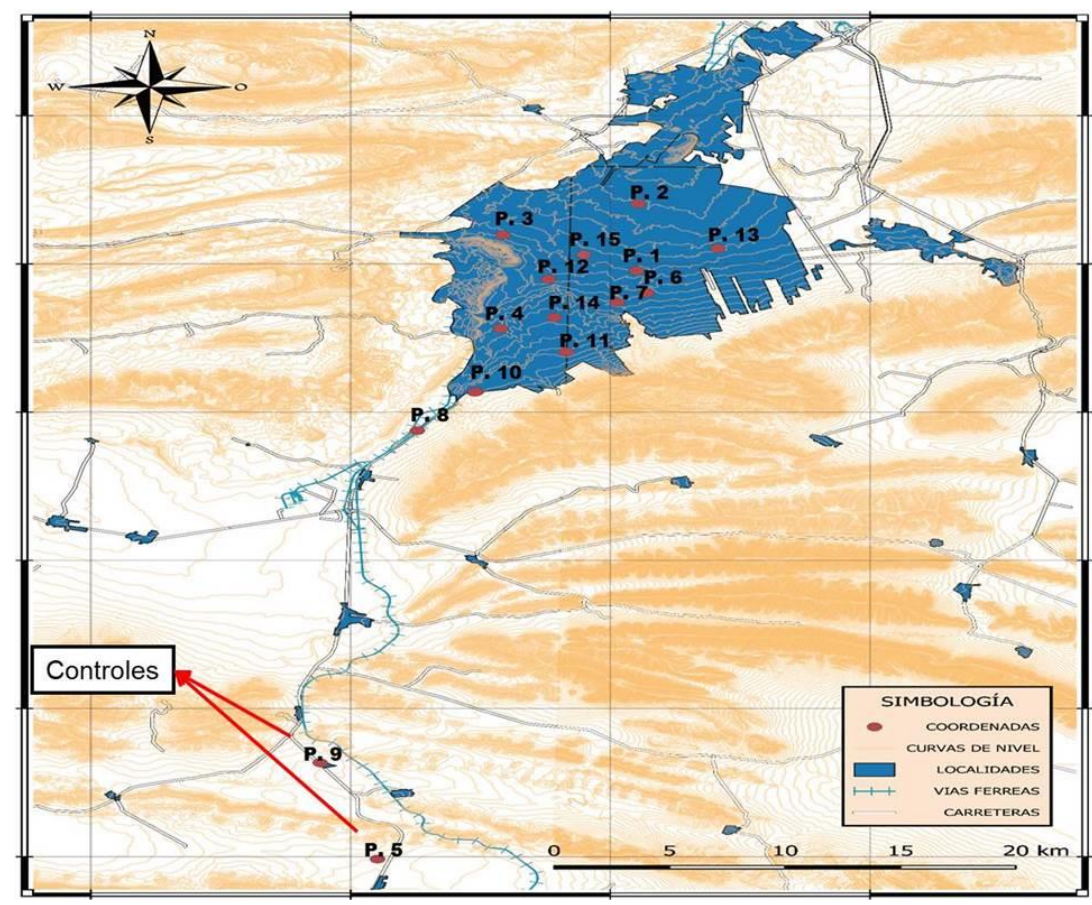

Figura 1. Área de estudio y distribución de los puntos de muestreo. 
Se colectaron $100 \mathrm{~g}$ de material foliar tomada a $2.5 \mathrm{~m}$ de altura, se lavaron con agua destilada varias veces y luego con agua desionizada. Las muestras fueron secadas en horno de secado a temperatura constante de $80{ }^{\circ} \mathrm{C}$ hasta alcanzar un peso constante. Se tomaron $2 \mathrm{~g}$ de muestra macerada y se colocaron en una mufla a una temperatura de $600{ }^{\circ} \mathrm{C}$ por $3 \mathrm{~h}$. Una vez obtenidas las cenizas, se realizó la digestión con $10 \mathrm{ml}$ de $\mathrm{HCL}_{\text {y }} \mathrm{HNO}_{3}$ (en proporción 1:3) en parrilla eléctrica durante 1520 minutos.

La muestra fue aforada a $25 \mathrm{ml}$ con agua desionizada y luego fue filtrada con papel Wathman \#41 para realizar el análisis de los elementos. La determinación de los metales pesados se realizó a través de un equipo espectrometría de emisión óptica de plasma inductivamente acoplado (ICPOES), Modelo Varian 730-ES.

\section{Resultados y discusión}

De acuerdo con los resultados obtenidos de los análisis de $\mathrm{MP}(\mathrm{Cd}, \mathrm{Cr}, \mathrm{Pb}$ y $\mathrm{V})$ en las hojas de las especies arbóreas seleccionadas se pudo observar que el único punto con presencia de $\mathrm{Cd}$ fue el 4 $\left(54.57 \mu \mathrm{g} \mathrm{g}^{-1}\right)$ en la especie $C$. sempervirens (Figura 2). Para el Cr la especie que presentó mayor contenido fue $P$. halepensis en los puntos $2\left(60.52 \mu \mathrm{g} \mathrm{g}^{-1}\right), 3\left(75.74 \mu \mathrm{g} \mathrm{g}^{-1}\right), 6\left(68.6 \mu \mathrm{g} \mathrm{g}^{-1}\right), 10$ $\left(76.95 \mu \mathrm{g} \mathrm{g}^{-1}\right), 11\left(54.21 \mu \mathrm{g} \mathrm{g}^{-1}\right), 12\left(64.33 \mu \mathrm{g} \mathrm{g}^{-1}\right), 13\left(57.53 \mu \mathrm{g} \mathrm{g}^{-1}\right), 14\left(54.46 \mu \mathrm{g} \mathrm{g}^{-1}\right)$ y $15(74.03$ $\left.\mu \mathrm{g} \mathrm{g}^{-1}\right)$. La especie $C$. sempervirens presentó alta concentración en los puntos $3\left(63.34 \mu \mathrm{g} \mathrm{g}^{-1}\right), 4$ $\left(129.29 \mu \mathrm{g} \mathrm{g}^{-1}\right), 12\left(76.28 \mu \mathrm{g} \mathrm{g}^{-1}\right)$ y $15\left(63.93 \mu \mathrm{g} \mathrm{g}^{-1}\right)$ y Prosopis spp., sólo en el punto 12 (69.87 $\mu \mathrm{g} \mathrm{g}^{-1}$ ) (Figura 3).

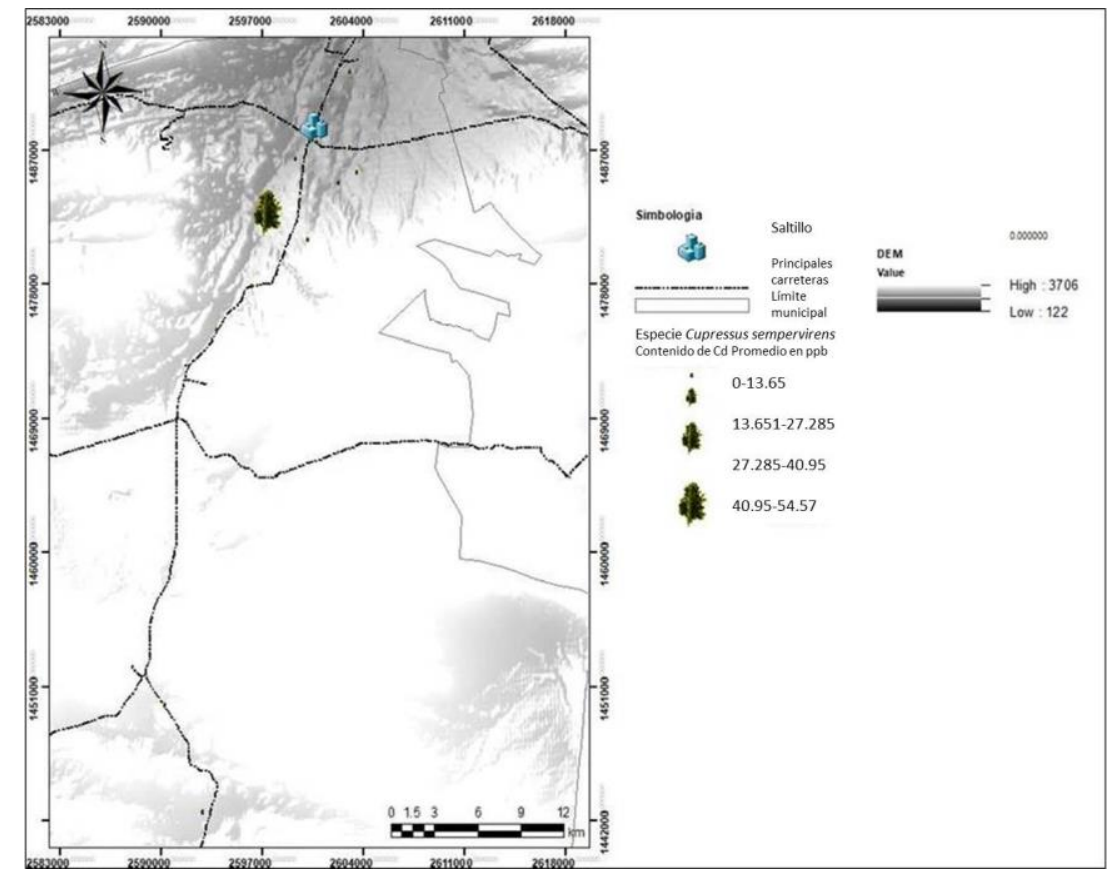

Figura 2. Contenido de cadmio (Cd) en los diferentes puntos de muestreo evaluados. 


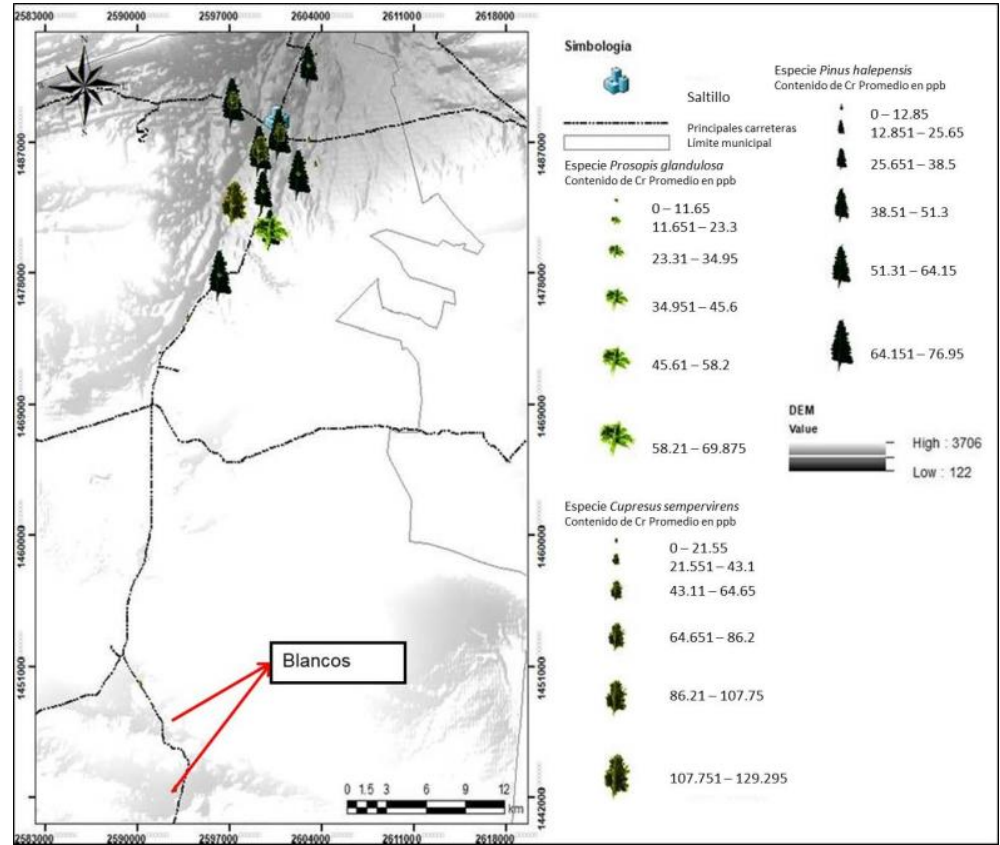

Figura 3. Contenido de cromo ( $\mathrm{Cr}$ ) en los diferentes puntos de muestreo evaluados.

El Pb solo se encontró en los puntos $4\left(783.67 \mu \mathrm{g} \mathrm{g}^{-1}\right)$ y $15\left(119.54 \mu \mathrm{g} \mathrm{g}^{-1}\right)$ en la especie $C$. serpenvirens y en el punto 2 (138.26 $\mu \mathrm{g} \mathrm{g}^{-1}$ ) en Prosopis spp. (Figura 4). La concentración de V fue mayor en la especie $C$. sempervirens en los puntos $3\left(59.48 \mu \mathrm{g} \mathrm{g}^{-1}\right)$ y $4\left(115.99 \mu \mathrm{g} \mathrm{g}^{-1}\right)$. Pinus halepensis presentó V solo en el punto $14\left(51.92 \mu \mathrm{g} \mathrm{g}^{-1}\right)$. Prosopis spp. sólo en el punto 4 (54.23 $\mu \mathrm{g} \mathrm{g}^{-1}$ ) (Figura 5).

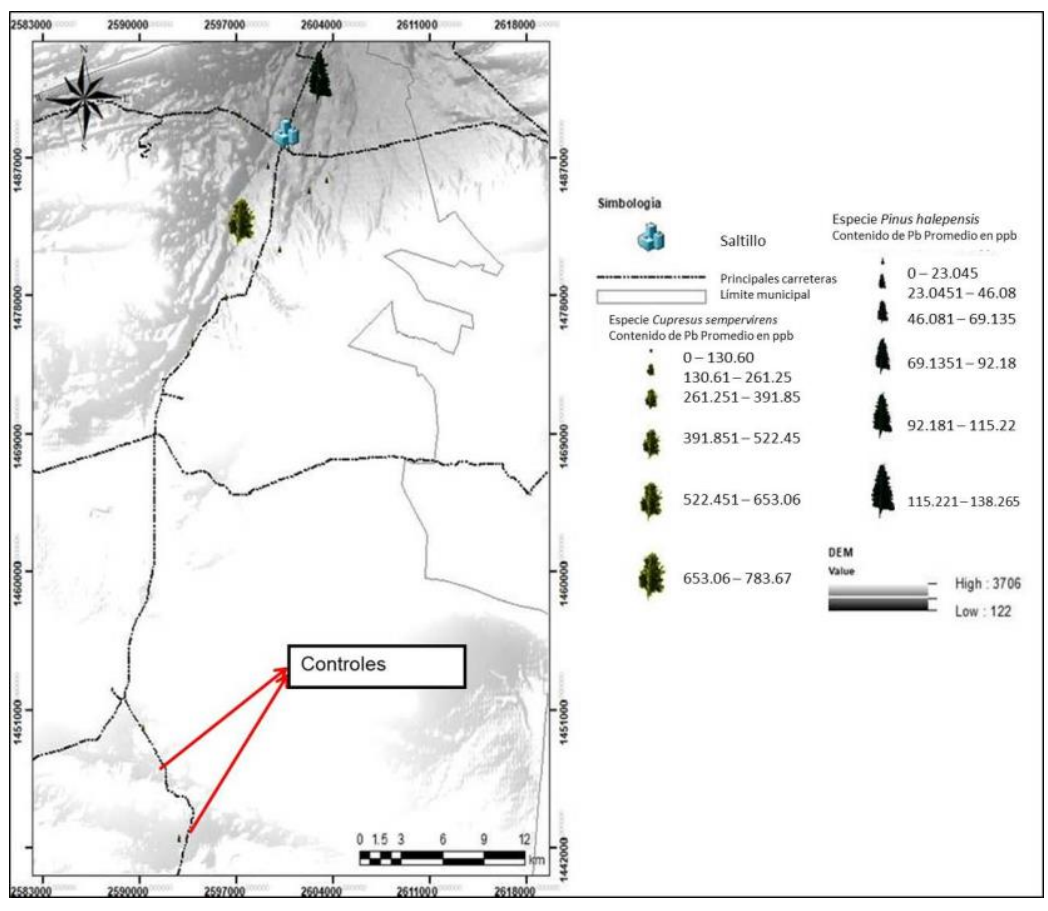

Figura 4. Contenido de plomo (Pb) en los diferentes puntos de muestreo evaluados. 


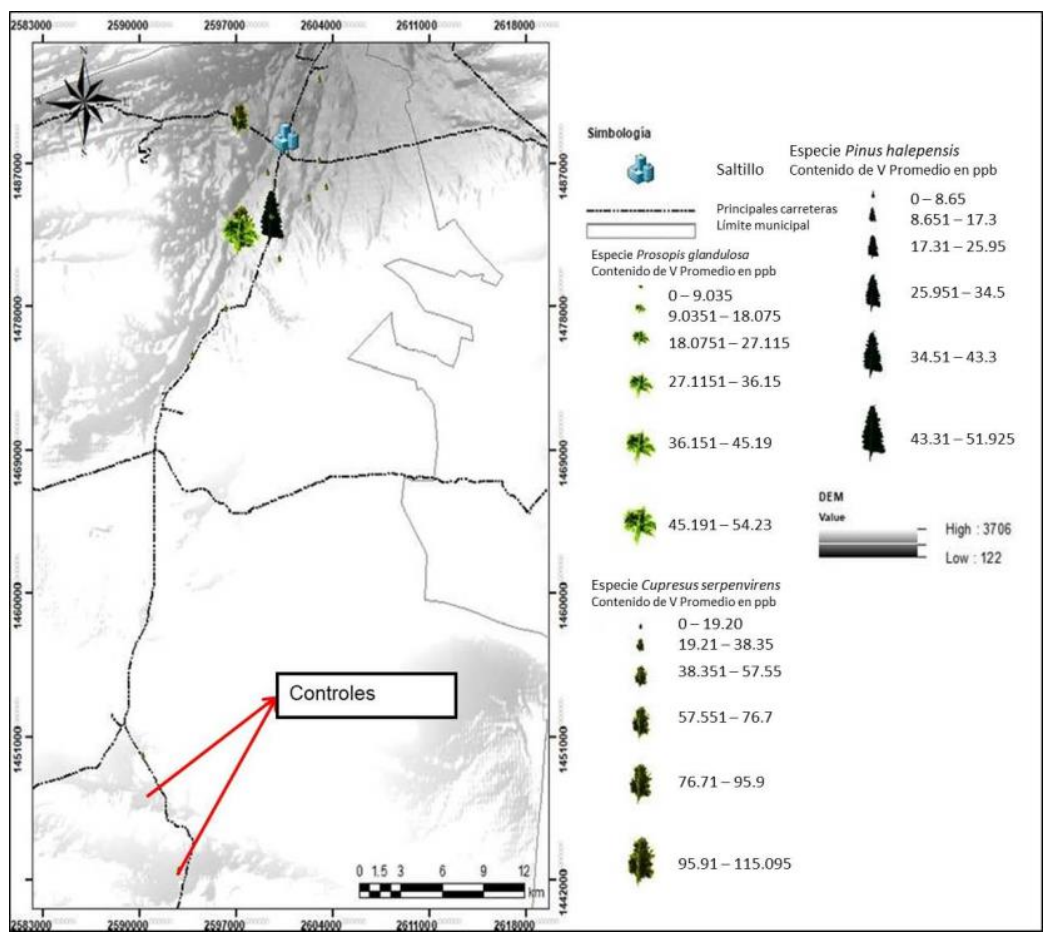

Figura 5. Contenido de vanadio (V) en los diferentes puntos de muestreo evaluados.

De acuerdo con los resultados, la especie $C$. sempervirens resultó la de mayor concentración de $\mathrm{Cd}, \mathrm{Pb}$ y V, mientras que $P$. halepensis fue la de mayor concentración de Cr. Esto demuestra que las especies de hojas perennes pueden concentrar metales pesados. Los puntos de control no presentaron ninguna concentración de metales pesados, a diferencia de los puntos ubicados en la ciudad.

En la actualidad los entornos urbanos pueden representar mayor exposición de contaminación por MP que sitios polvorientos (Alcalá et al., 2012) y que las zonas industriales tienen una marcada influencia de estos contaminantes en las ciudades (Aragón-Piña et al., 2006). El municipio de Ramos Arizpe cuenta con una de las zonas industriales más importantes y grandes del estado de Coahuila (Mendoza, 2001). Por lo que dicha industria pudiera estar aportando algunos contaminantes que pudieran estar siendo arrastrados por la dirección del viento y quedar retenidos en algunos puntos de la ciudad de Saltillo.

Investigaciones de Herrera-Márquez (2002), reportó en su estudio de calidad del agua a lo largo del 'Arroyo del Pueblo' en la ciudad de Saltillo, concentraciones promedio de $\mathrm{Pb}$ de $20 \mu \mathrm{g} \mathrm{L}{ }^{-1}$ (época de invierno). Mientras que en este trabajo se encontraron valores de $783.67 \mu \mathrm{g} \mathrm{g}^{-1}$ (punto 4) y $119.54 \mu \mathrm{g} \mathrm{g}^{-1}$ (punto 15) en la especie C. serpenvirens y $138.26 \mu \mathrm{g} \mathrm{G}^{-1}$ (Punto 2) en Prosopis spp., para este elemento.

Autores como Ramírez-Leal, (1986) mediante el análisis de muestreados de alto volumen, para el año 1984 las principales concentraciones de plomo $\left(0.217 \mu \mathrm{g} \mathrm{m}^{-3}\right)$ y cromo $\left(0.029 \mu \mathrm{g} \mathrm{m}^{-3}\right)$ fueron en el mes marzo y la de cadmio $\left(0.037 \mu \mathrm{g} \mathrm{m}^{-3}\right)$ en octubre. Para el periodo de 1985 los valores más altos de cadmio $\left(0.032 \mu \mathrm{g} \mathrm{m}^{-3}\right)$ se registraron en el mes de octubre, de plomo $\left(0.13 \mu \mathrm{g} \mathrm{m}^{-3}\right)$ en julio 
$\left(63.69 \mu \mathrm{g} \mathrm{m}^{-3}\right)$ y la de cromo $\left(0.155 \mu \mathrm{g} \mathrm{m}^{-3}\right)$ en abril. Esto puede indicar que existe variación de los metales pesados dependiendo de la temporada, ya que son varias las condiciones que determinan las concentraciones de estos elementos a través del tiempo.

La vegetación arbórea, además de embellecer los escenarios de las ciudades, mejora la calidad del aire significativamente, ya que remueve las partículas suspendidas en el ambiente, lo cual es un servicio ecológico importante. La capacidad de retención de contaminantes en sus copas es posible usarla como un indicador de calidad ambiental (Astorga-Bustillos et al., 2011).

Al respecto, existen diversos trabajos de investigación con diferentes tipos de plantas y se ha determinado que las hojas fasciculadas de pino son el material más utilizado, junto con los musgos, para este propósito (Piccardo et al., 2005), entre las especies de pinos con las que se han realizado estudios de biomonitoreo ambiental por contaminantes, se encuentran $P$. sylvestris (Shcherbenko et al., 2008), P. eldarica (Kord et al., 2010), P. halepensis (Astorga-Bustillos et al., 2011), P. pinea (Rucandio et al., 2011). Sin embargo, las cupresáceas también han demostrado ser especies bioindicadoras de contaminación ambiental, algunas de las especies con las que se ha trabajado son Cupressus arizonica (Alcalá et al., 2008) y C. sempervirens (Rucandio et al., 2011).

Una ventaja de las coníferas sobre las especies de hoja ancha (hojas caducifolias) es que acumulan contaminantes atmosféricos por varios años por el hecho de ser especies de hojas perennes (Di Guardo et al., 2003). Sin embargo, a pesar de que las especies con hojas perennes son útiles para tal fin, la interpretación cuantitativa de los datos es complicada por la falta de estudios comparativos (Hellström et al., 2004).

\section{Conclusiones}

Las zonas sureste y céntrica de la ciudad de Saltillo presentan las mayores concentraciones de los MP analizados $(\mathrm{Cd}, \mathrm{Cr}, \mathrm{Pb}$ y $\mathrm{V})$. La especie Cupressus sempervirens resulto la de mayor concentración de $\mathrm{Cd}, \mathrm{Pb}$ y V, mientras que la especie Pinus halepensis fue mayor en $\mathrm{Cr}$. Lo anterior, demuestra que las especies perennifolias pueden acumular metales pesados en el tejido foliar, lo que indica que puede ser utilizadas como especies para el biomonitoreo de contaminación por metales pesados.

Los puntos de control no tuvieron ningún nivel de concentración de los metales pesados analizados, lo que muestra que en la ciudad de Saltillo pueden existir fuentes de contaminantes que provocan la aparición de algunos de estos elementos.

\section{Literatura citada}

Alcalá, J.; Sosa, M.; Moreno, M.; Quintana, C.; Quintana, G.; Miranda, S. y Rubio, A. 2008. Metales pesados en vegetación arbórea como indicador de la calidad ambiental urbana: ciudad de Chihuahua, México. Multequina. 17:39-54.

Alcalá-Jáuregui, J.; Ávila-Castorena, C.; Rodríguez-Ortíz, J. C.; Hernández-Montoya, A.; BeltránMorales, F. A.; Rodríguez-Fuentes, H. y Loya-Ramírez, J. G. 2012. Metales pesados como indicador de impacto de un sistema ecológico fragmentado por usos de suelo, San Luis Potosí, México. Rev. Facul. de Cienc. Agrar. 44(2):15-29. 
Aragón-Piña, A.; Campos-Ramos, A. A.; Leyva-Ramos, R.; Hernández-Orta, M.; MirandaOrtiz, N. y Luszczewski-Kudra, A. 2006. Influencia de emisiones industriales en el polvo atmosférico de la ciudad de San Luis Potosí, México. Rev. Int. Contam. Ambient. 22(1):5-15.

Astorga, B.; Fernando, R.; Sosa C.; Manuel, H. P.; Eduardo, F.; Moreno, L.; Myriam, V.; Tena, V.; Melitón, C. y Trujillo, A. 2011. Pinus halepensis Mill: como indicador de contaminación atmosférica en una zona industrial urbana. Rev. Mex. Cienc. Forest. 2(7):79-86.

Covarrubias, S. A. y Cabriales, J. J. P. 2017. Contaminación ambiental por metales pesados en México: Problemática y estrategias de fitorremediación. Rev. Intern. Contam. Ambient. 33(Esp. 1):7-21.

Cruz-Campas, M. E.; Gómez-Álvarez, A.; Quintero-Núñez, M. y Varela-Salazar, J. 2013. Evaluación de la calidad del aire respecto de partículas suspendidas totales (PST) y metales pesados $(\mathrm{Pb}, \mathrm{Cd}, \mathrm{Ni}, \mathrm{Cu}, \mathrm{Cr})$ en la Ciudad de Hermosillo, Sonora, México, durante un periodo anual. Rev. Intern. Contam. Ambient. 29(4):269-283.

Di-Guardo, A.; Zaccara, S.; Cerabolini, B.; Acciarri, M.; Terzaghi, G. and Calamari, D. 2003. Conifer needles as passive biomonitors of the spatial and temporal distribution of DDT from a point source. Chemosphere. 52(5):789-797.

Fernández, A.; Ternero, M.; Barragán, F. and Jiménez, J. 2000. An approach to characterization of sources of urban airborne particles through heavy metal speciation. Chemosphere-Global Change Sci. 2(2):123-136.

González-Chávez, M. C. A.; Carrillo-González, R.; Sánchez-López, A. S. y Ruiz-Olivares, A. 2017. Alternativas de fitorremediación de sitios contaminados con elementos potencialmente tóxicos. Agroproductividad. 10(4):8-14.

Henry, J. G. y Heinke, G. W. 1999. Ingeniería ambiental. Segunda edición. Editorial Pearson Educación. 778 p.

Hellström, A. H.; Kylin, W.; Strachan, M. J. and Jensen, S. 2004. Distribution of some organochlorine compounds in pine needles from Central and Northern Europe. Environmental Pollution. 128(1-2):29-48.

Hernández-Bárcenas, G.; Castillo, F.; Ávalos-Borja, M. y Martínez-Villegas, N. V. 2017. Síntesis de arseniatos de calcio (guerinita, haidingerita y farmacolita) morfológicamente similares a los encontrados en suelos contaminados. Rev. Int. Contam. Ambient. 33(1):153-163.

Herrera, M. 2002. Determinación de indicadores de calidad del agua en el arroyo "del pueblo" de Saltillo, Coahuila. Tesis de Licenciatura. Universidad Autónoma Agraria Antonio Narro. $93 \mathrm{p}$.

INEGI. 2015. Instituto Nacional de Estadística y Geografía. Principales resultados de las encuestas intercensal. Coahuila de Zaragoza. México.

Kumar, P. R. S.; Lee, S.; Zhang, M.; Fai, Y.; Tsang, K. and Kim, H. 2019. Heavy metals in food crops: Health risks, fate, mechanisms, and management. Environ Inter. 125:365-385.

Liu, G.; Wang, J.; Liu, X.; Liu, X.; Li, X.; Ren, Y.; Wang, J.; and Dong, L. 2018. Partitioning and geochemical fractions of heavy metals from geogenic and anthropogenic sources in various soil particle size fractions. Geoderma. 312:104-113.

Kord, B.; Mataji A. and Babaie, S. 2010. Pine (Pinus eldarica Medw.) needles as indicator for heavy metals pollution. International J. Environ. Sci. Technol. 7(1):79-84.

Mendoza, J. 2001. Crecimiento y especialización en la región Saltillo-Ramos Arizpe. Comercio Exterior. 51(3):250-258. 
Piccardo, M. T.; Pala, M.; Bonaccurso, B.; Stella, A.; Redaelli, A.; Paola, G. and Valerio, F. 2005. Pinus nigra and Pinus pinaster needles as passive samplers of polycyclic aromatic hydrocarbons. Environ. Pollution. 133(2):293-301.

Ramírez-Leal, R. 1986. Estudio preliminar de evaluación de la calidad del aire, en la Ciudad de Saltillo, Coahuila, México. Tesis de Maestría. Universidad Autónoma de Nuevo León. 55 p.

Rodríguez-Martínez, H. L.; Peña-Manjarrez, M.; Gutiérrez-Reyes, A. V.; González-Trevizo, C. L.; Montes-Fonseca, S. L. y López-Avalos, G. G. 2017. Biorremediación de arsénico mediada por microorganismos genéticamente modificados. Terra Latinoam. 35(4):353-361.

Rucandio, M. I.; Petit-Domínguez, M. D.; Fidalgo-Hijano, C. and García-Giménez, R. 2011. Biomonitoring of chemical element in an urban environment using arboreal and bush plant species. Environ. Sien. Pollution Res. 18:51-63.

SEMA. 2017. Secretaría de Medio Ambiente. Programa de gestión para mejorar la calidad del aire del estado de Coahuila de Zaragoza. 2017-2026 pp.

Shcherbenko, T. A.; Koptsik, G. N.; Groenenberg, B. J.; Lukina, N. V. and Livantsova, S. Y. 2008. Uptake of nutrients and heavy metals by pine trees under atmospheric pollution. Moscow University. Soil Science Bulletin. 63:51-59.

Turkyilmaz, A.; Sevik, H. and Cetin, M. 2018. The use of perennial needles as biomonitors for recently accumulated heavy metals. Landscape Ecol. Eng. 14:115-120. 